\title{
Comparison of Morphology-Based and Delay-Based Measures for Reference Beat Classification during Atrial Tachycardia
}

\author{
Laura Anna Unger ${ }^{1}$, Armin Luik $^{2}$, Annika Haas ${ }^{2}$, Olaf Dössel ${ }^{1}$ \\ ${ }^{1}$ Karlsruhe Institute of Technology - Insititute of Biomedical Engineering, Karlsruhe, Germany \\ ${ }^{2}$ Städtisches Klinikum Karlsruhe - Medizinische Klinik IV, Karlsruhe, Germany
}

\begin{abstract}
Beat acceptance and rejection during atrial tachycardia are crucial for the compilation of meaningful maps during an electrophysiological study. While state of the art methods compare the delays in activation time between two or more reference electrograms, morphology based beat selection might enhance the quality of the resulting map.

This work introduces morphology-based measures for beat selection during atrial tachycardia in a spatially stable reference electrogram and compares them to delaybased measures.

In an exemplary patient, the introduced measures proved to be complementary. Using an optimized combination of morphology- and delay-based beat selection criteria will therefore be beneficial for automatic beat selection in electroanatomical mapping systems.
\end{abstract}

\section{Introduction}

Atrial tachycardia (AT) is a cardiovascular condition of high prevalence and typically indicates an electrophysiological study to diagnose and treat the individual patient. A three-dimensional electroanatomical mapping system acquires a sequential ultra-high-resolution map of the repetitive AT and helps the physician to understand the underlying mechanism and to deduce the optimal treatment strategy. An electroanatomical map of high accuracy with reliable details serves as the basis for all following therapeutic decisions. Therefore, it is crucial to forward only representative beats to the map.

All state of the art electroanatomical mapping systems feature semi-automatic or automatic beat selection tools. A spatially stable reference catheter in the coronary sinus (CS) acquires two or more reference electrograms (EGMs) [1]. After calculating the reference activation times from the reference EGMs, a beat presumably belongs to the target AT if the time delays between the reference activation times are within a preset range.

This work focuses on the assessment of different meth- ods to distinguish beats belonging to the target arrhythmia from distorting beats. The latter can be of various origin including atrial extrasystoles or changes in the AT. The clinical state of the art commonly bases the selection of target beats on the time delay between two reference activation times. Delays between several reference activation times can optionally be taken into account. This work also includes the assessment of morphological similarity between reference EGMs for beat selection and compares advantages and drawbacks of the respective approaches.

\section{Methods}

\subsection{Dataset}

For an exemplary patient, the clinically dominant AT was mapped with the electroanatomical mapping system Rhythmia HDx (Boston Scientific). Surface electrocardiograms (ECGs) and bipolar intracardiac EGMs measured with the CS catheter were imported to MATLAB for retrospective analysis. Beat acceptance criteria as defined by the mapping system were not taken into account.

\subsection{Active Segments}

In a first step, activity packages were highlighted individually for each of the typically seven bipolar CS leads by thresholding the non-linear energy operator $N L E O=x_{n}^{2}-x_{n-1} x_{n+1}$ of a discrete EGM $x$ [2]. In order to eliminate artifacts in individual bipolar CS leads, a temporal overlap with active segments in at least three other bipolar CS leads was demanded for acceptance.

In a next step, local activation times (LATs) were determined for each of the active segments. Following the Rhythmia HDx system, the maximum and the minimum voltage of each active segment defined two sets of LATs. To decide for the most suitable set of LATs for each CS lead, the cycle length was calculated as time delay between subsequent LATs. Cycle lengths below and above the $5 \%$ and $95 \%$ quantile were excluded from the series. The set of LATs yielding cycle lengths of lower standard deviation 
was considered most suitable for the respective bipolar CS lead.

\subsection{Ventricular Overlap}

As the CS catheter is located close to the junction between atria and ventricles, the acquired signal contains components of both the atria and the ventricles. The impact of the ventricular activation on the CS EGM varies among the individual bipolar electrode combinations. In order to be able to distinguish between active segments with ventricular components and those with plain atrial activity, the detection of QRS complexes in the surface ECG served as a reference.

\subsection{Best CS leads}

In order to reconstruct the clinical scenario of using the time delay between two most suitable CS leads as beat acceptance criterion, the latter needed to be defined. In this work, the standard deviation of cycle lengths after exclusion of outliers as described above measured the quality of a CS EGM. The bipolar lead combination of lowest standard deviation in cycle length was considered the most suitable CS lead. The second and third most suitable CS lead was found accordingly.

\subsection{Clustering by Beats}

For a beat based analysis, active segments were clustered by beats. Starting with the most distal lead and proceeding in proximal direction, an active segment was added to an existing beat if its LAT was located in proximity to active segments that had already been assigned to the beat. The proximity interval was set to begin a quarter of the cycle length to the left of the earliest begin of an active segment within the beat and was set to end a quarter of the cycle length to the right of the latest end of an active segment within the beat.

If an incoming active segment did not match this criterion for any of the existing beats, a new beat was compiled.

\subsection{Measures of Beat Acceptance}

This work introduces and compares three measures to distinguish between target beats and flawed beats.

The most commonly used measure in clinical practice is the time delay $\Delta R$ between the LATs of the two most suitable CS leads:

$$
\Delta R=L A T_{I}-L A T_{I I}
$$

with I and II denoting the most suitable and second most suitable bipolar CS lead. Alternatively, the time delay between LATs of several lead combinations can be consid- ered. In this case, stacking several realizations of $\Delta R$ results int the vector $\overrightarrow{\Delta R}$,

The second and third measure measure the morphological similarity of two active segments. Any pair of active segments within the same CS lead was prealigned according to the maximum of the non-normalized crosscorrelation $\Phi_{x y}$ :

$$
\Phi_{x y}\left(k T_{a}\right)=\sum_{n=1}^{N} x_{n} \cdot y_{n-k}
$$

with $\mathrm{N}$ being the length of active segment $x$ and $k \in$ $[-M+1 ; M-1]$ while $M$ is the length of active segment $y . k T_{a}$ defines the time lag consisting of $k$ samples of length $T_{a}$.

Both active segments were cut to the overlapping samples yielding the preprocessed active segments $\tilde{x}$ and $\tilde{y}$ of length $L$.

The morphological similarity was defined by two different measures:

1. Correlation based similarity: The correlation coefficient $\rho_{\tilde{x} \tilde{y}}$ yields a similarity measure between -1 and 1 :

$$
\rho_{\tilde{x} \tilde{y}}=\frac{1}{L-1} \sum_{i=1}^{L} \frac{\tilde{x}_{i}-\mu_{\tilde{x}}}{\sigma_{\tilde{x}}} \cdot \frac{\tilde{y}_{i}-\mu_{\tilde{y}}}{\sigma_{\tilde{y}}}
$$

$\tilde{x}_{i}$ and $\tilde{y}_{i}$ denote the $i$-th sample of the respective active segment.

2. Distance based similarity: The normalized 1-norm distance $d_{\tilde{x} \tilde{y}}$ between a pair of preprocessed active segments is defined as

$$
d_{\tilde{x} \tilde{y}}=\frac{1}{L} \cdot \sum_{i=1}^{L}\left|\tilde{x}_{i}-\tilde{y}_{i}\right|
$$

\section{Results}

\subsection{Time Delay between Beats}

Fig. 1 shows the time delays between LATs of different bipolar CS leads. CS lead 7-8 was determined to be the most suitable lead for LAT detection. Therefore, all other delays were referenced to this lead. The second and third most suitable leads were CS leads 6-7 and 3-4, respectively. While the LAT delay between lead 6-7 and lead 7-8 was negligible, the delay was longer for the other leads and jumped at beat 2319. Bipolar leads 1-2 and 2-3 were overlapped by the ventricular field and were thus least suitable for the calculation of delays. The wide spread of values in the uppermost panels of Fig. 1 visualizes this context and emphasizes that the suitability of $\Delta R$ as beat selection criterion is strongly dependent on both the quality of EGMs and the selection of suitable pairs. 

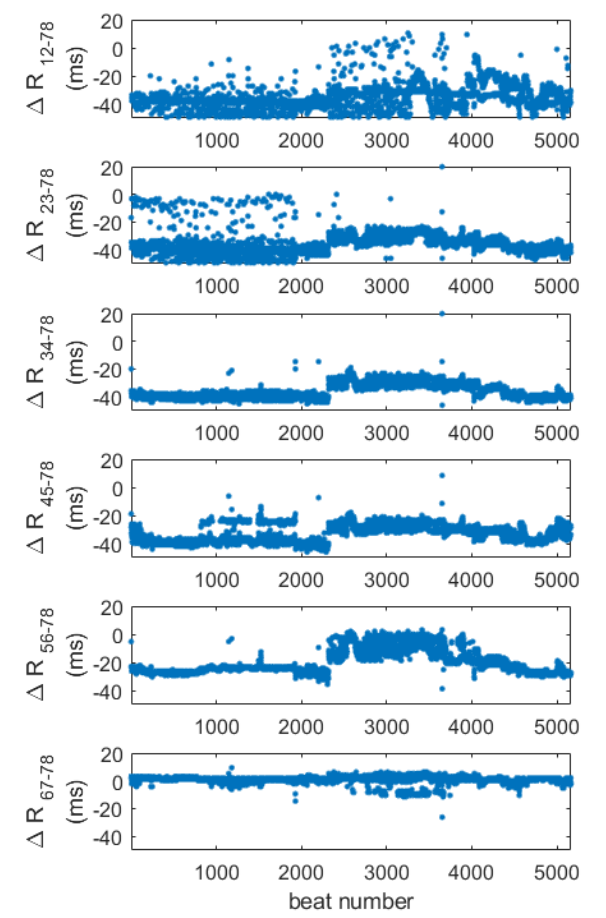

Figure 1: Time delay measured between the LATs of two bipolar CS leads. CS lead 7-8 was considered the reference channel while the other bipole alternates between the subplots as indicated on the y-axis.

\subsection{Morphological Similarity}

Fig. 2 shows the correlation based similarity measure between any two beats of the exemplary target map. The main diagonal represents the auto-correlation of an active segment with itself and thus equals 1 . Squares in similar shades of yellow along the main diagonal cluster subsequent beats of similar morphology. A small yellow square ranging from beat 1926 to beat 2319 is separated from the other beats by two broad green bars of low correlation. Those isolated beats are similar among each other but dissimilar to preceding and succeeding beats. Most likely, those beats do not represent the target AT. Fig. 3 displays the corresponding EGMs. While beat 1918 through beat 1925 are highly correlated and similar in morphology with exception of beat 1922 and beat 1924, the morphology of beat 1926 and following differs significantly. Beat 1931 through 1940 are similar among themselves but not to the preceding beats as previously outlined in the correlation grid in Fig. 2. While the end of the beats of differing morphology was already indicated by a jump in $\Delta R$ (see Fig. 1), $\Delta R$ was blind to the beginning of the sequence.

Fig. 4 displays the similarity measured by means of the normalized 1-norm distance $d$. While the basic grid characteristics are comparable to the correlation-based similar-

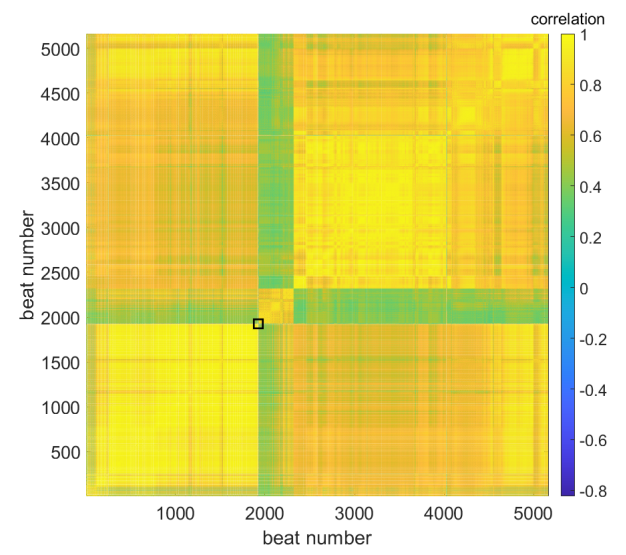

(a) All beats recorded during mapping of the target AT. The black rectangle indicates the window of interest for (b).

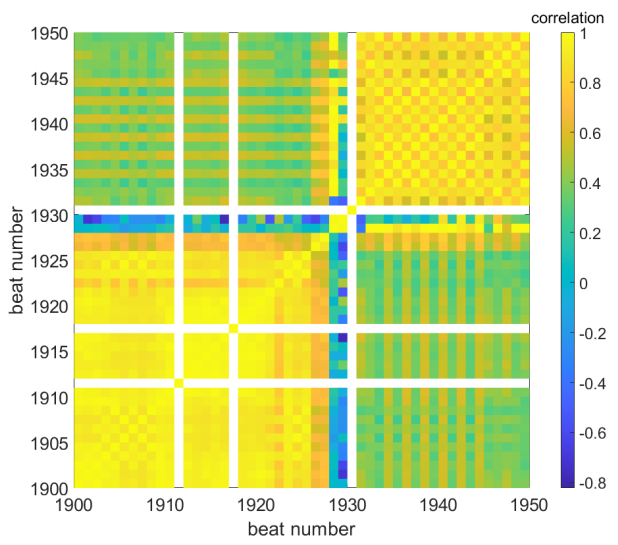

(b) Window of interest ranging from beat 1900 to beat 1950 . Beats without activity in the CS lead 7-8 are displayed in white.

Figure 2: Morphological similarity based on the correlation of reference EGMs recorded with CS bipolar lead 7-8.

ity, the two measures stress deviations in morphology differently.

The interference of atrial and ventricular components in the active segments was reflected in alternating morphological similarity. The exemplary patient presented with a 2:1 AT, i.e. every other atrial beat was overlapped by a ventricular contraction. Fig. 2 (b) is therefore overlaid by a checkered pattern. The analysis of morphological similarity in other bipolar CS leads showed stronger checkered patterns for the more distal CS leads.

\section{Discussion and Outlook}

Each measure for beat acceptance comes with individual advantages and disadvantages. While $\Delta R$ is easily computable, several different ATs can result in similar values, which leads to misclassification of beats. Taking into account several time delays $\overrightarrow{\Delta R}$ can improve the accuracy. However, the reliability of time delays strongly depends 

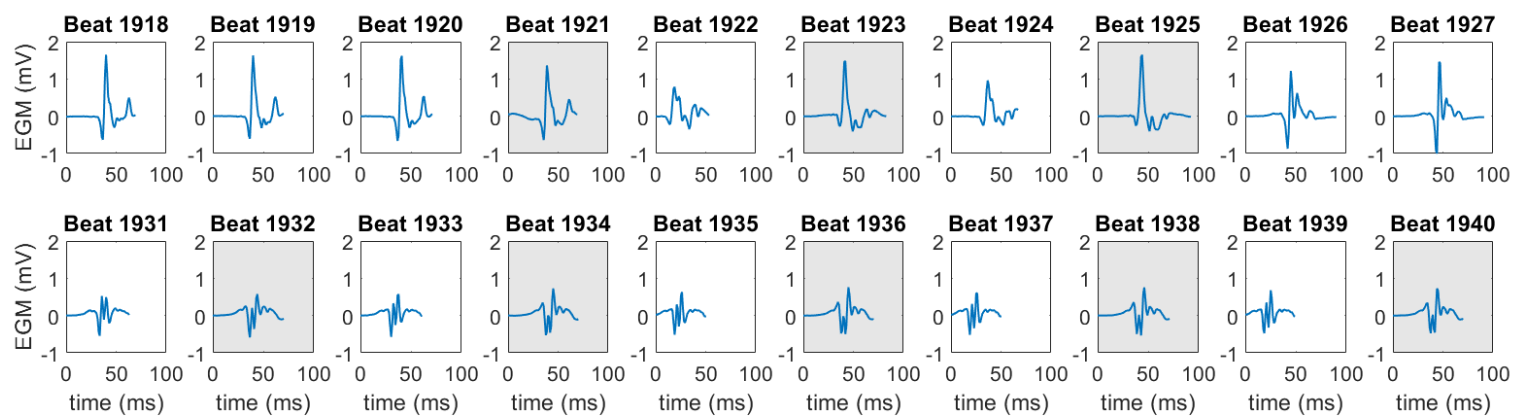

Figure 3: Active segments recorded with bipolar CS lead 7-8. Gray background indicates an overlapping QRS complex. Compare Fig. 2 (b) for individual beat correlations.

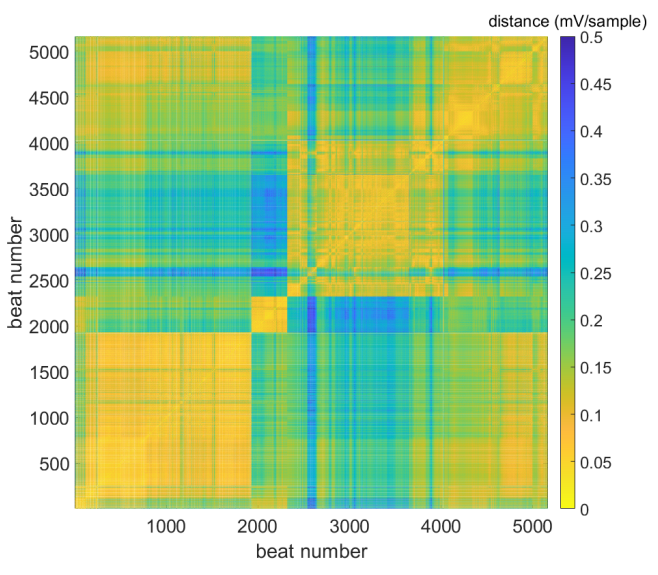

Figure 4: Morphological similarity based on the distance of reference EGMs recorded with CS bipolar lead 7-8.

on the determined LATs. In clinical practice, only a limited number of CS leads is suitable for reliable LAT assignments. For some patients, it might even be hard to define a minimum of two suitable CS leads.

Both morphology based features introduced in this work are independent from accurate LATs. Taking into account the morphology of the entire active segment advances beat selection. However, global changes in conduction velocity due to pharmacological administrations or other influencing factors are hardly detectable by morphological measures if the excitation pathways are maintained. This drawback could be overcome by additionally taking into account $\Delta R$.

Comparing the distance-based and the correlation-based morphological measures, $\rho_{\tilde{x} \tilde{y}}$ comes with the advantage of a specified range of possible values while $d_{\tilde{x} \tilde{y}}$ does not have an upper limit. On the other hand, $\rho_{\tilde{x} \tilde{y}}$ is independent from changes in amplitude and phase of the signal, which might result in inaccuracies that can be overcome by the distance-based measure.

A modified $\Delta R$ parameter could help to overcome un- certainties in LAT by replacing the LAT-based time delay by the correlation based time lag $k T_{a}$.

Besides beat selection, the introduced morphological features will also enhance the detection of changes in the ongoing AT under ablation. While a slowing of the AT is easily detectable by $\Delta R$, changes in morphology could also tackle varying excitation pathways.

While the introduced measures suggest that EGM morphology enhances beat selection for the exemplary patient, a larger study cohort covering a representative selection of AT patterns, atrial geometries, and substrate could quantify the achievable benefit in a follow-up study. A combined measure could optimize the contribution of the measures presented individually in this work.

\section{References}

[1] Kim YH, Chen SA, Ernst S, Guzman CE, Han S, Kalarus Z, Labadet C, Lin YJ, Lo LW, Nogami A, Saad EB, Sapp J, Sticherling C, Tilz R, Tung R, Kim YG, Stiles MK. 2019 APHRS expert consensus statement on three-dimensional mapping systems for tachycardia developed in collaboration with HRS, EHRA, and LAHRS. Journal of Arrhythmia 2020;36(2):215-270.

[2] Schilling C, Nguyen MP, Luik A, Schmitt C, Dössel O. Nonlinear energy operator for the analysis of intracardial electrograms. In IFMBE Proceedings World Congress on Medical Physics and Biomedical Engineering, volume 25/4. 2009; 872-875.

Address for correspondence:

Laura Anna Unger, M.Sc.

Institute of Biomedical Engineering

Karlsruhe Institute of Technology (KIT)

Fritz-Haber-Weg 1, 76131 Karlsruhe, Germany

publications@ibt.kit.edu 